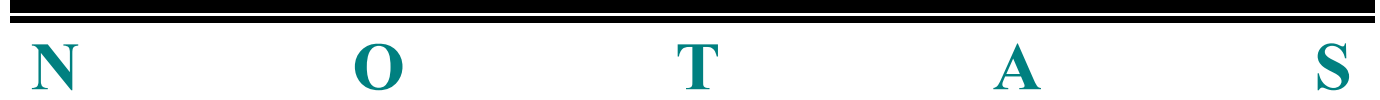




\section{NOTA SOBRE LA HISTORIA DE CLS EN LOS ESTADOS UNIDOS*}

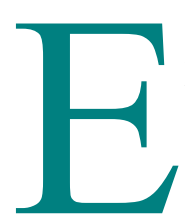

n sus comienzos, hacia 1977, CLS se constituye como una intervención, a la vez política e intelectual, en el «campo académico» [Bourdieu] de las facultades de Derecho, iniciada y renovada periódicamente por profesoras, profesores, estudiantas y estudiantes. Los temas políticos de la intervención son heterogéneos pero todos ellos de inclinación izquierdista. Los temas intelectuales son de tendencia «continental», con la importante excepción del realismo jurídico americano [Livingston, Schlegel, Gordon 1982, Binder, Tushnet 1991]. (Los artículos canadienses citados aquí forman parte integrante del desarrollo americano de CLS. Sobre la fortuna de CLS en Canadá, véase [Macklem]).

La intervención toma las siguientes formas: (1) la formación de «facciones de izquierda» en varias facultades; (2) la publicación en las revistas académicas de una nueva literatura jurídica; (3) la organización de «colonias de vacaciones» en las que algunas decenas de participantas y participantes discuten sus investigaciones y leen conjuntamente a los «clásicos»; (4) la celebración de grandes congresos en los que varios centenares de personas asisten a una multiplicidad de exposiciones de todas clases; (5) la constitución de una «red» nacional que apoye estas actividades.

En el plano intelectual, el trabajo más importante de los primeros años consiste en elaborar una crítica interna de la razón jurídica «american style» de los años 70. Esta crítica, producida por una veintena de autoras y autores y que no tienen, al parecer, equivalente europeo, se caracteriza por abordar detalladamente dominios jurídicos tales como los contratos [C. Dalton 1985, Mensch, Gordon 1987], el race law

* Este trabajo es la versión española de la voz «Critical Legal Studies» que aparecerá en la segunda edición, del «Dictionare de Theorie, du Droit» dirigido por André Arnaud. 
[Freeman 1978], el Derecho laboral [Klare 1978, Stone], el Derecho del bienestar [Simon] el Derecho penal [Kelman 1981], el Derecho de familia [Olsen 1983], el Derecho de la administración local [G. Frug 1980], el Derecho internacional [David Kennedy 1986], etc., y de las metodologías legitimantes que utilizan los juristas para apoyar y desarrollar estos dominios, tales como el positivismo liberal [Kelman 1987], el funcionalismo sociológico [Gordon 1984, Trubek], la doctrina de la interpretación constitucional [Tushnet 1988, Hutchinson y Petter, Bakan], etc.

La aspiración de la crítica es poner al descubierto el sentido político de la práctica cotidiana de los jueces y de los juristas, que construyen el Derecho mientras se ven a sí mismos como un instrumento del mismo. Algunos conceptos y palabras-clave son lagunas, conflictos y ambigüedades, indeterminación, mediación, legitimación del statu quo (pero no del «capitalismo»), estructuración jurídica de la base material (y en consecuencia imposibilidad de «determinación en la instancia final»). La actitud respecto al Derecho es ambivalente. Por un lado, se insiste en su autonomía relativa y en su valor en tanto que dominio de lucha cultural y política; por otro, se persigue con obstinación de mostrar su incoherencia, sus contradicciones internas, sus complicidades [Duncan Kennedy 1979, Katz, klare 1979, Tushnet 1982]. Al mismo tiempo que se toma en serio la noción neo-marxista de que el discurso jurídico equivale a ideología, hay una rebelión contra las categorías familiares y contra el tono del discurso izquierdista americano de la época, sobre todo contra su moralismo simplista [Gabel y Harris, Olsen 1990, Duncan Kennedy 1976, Unger 1986, David Kenney 1985].

La crítica CLS apunta «al interior» del Derecho, al dominio de las reglas detalladas, de los argumentos comunes, de las prácticas familiares de la producción académica y judicial. Los miembros del movimiento enseñan, por otra parte, preferentemente las materias elementales, y se ocupan con frecuencia en sus escritos de las cuestiones técnicas que preocupan a las corrientes dominantes. Es precisamente esto lo que les permite tener un efecto en las facultades (véase, como contraste, la marginalización de CLS en Europa).

Con pocas excepciones [Abel] no se trata aquí ni de filosofía, ni de sociología del Derecho, aunque se tome en préstamo sin pudor a los textos clásicos de estas disciplinas, de forma ecléctica, y aunque se pretenda separarlos mientras se los canibaliza. Esta inversión de las jerarquías convencionales de las disciplinas ocasiona muchos malentendidos, en parte porque sus motivaciones culturales y políticas son ininteligibles o repugnantes para la mayor parte de los filósofos y sociólogos profesionales. Su análogo europeo se encuentra, quizá, en 
la «crítica cultural (p. ej. Gramsci, Nietzsche, Benjamín), cuyas pretensiones filosóficas siempre han generado burla o irritación entre los profesionales. Por contraste, proliferan los estudios históricos y semióticos [Horwitz 1977, 1992, Vandevelde, Nockleby, Steinfeld, Peritz, y véanse bibliografías en Duncan Kennedy 1991].

Los «críticos» de esta época emprenden varias iniciativas sindicales en las facultades de Derecho. La gran mayoría se opone (1) al carácter reaccionario de la pedagogía de sus colegas, (2) a los procedimientos y criterios que limitan el acceso de las mujeres y de las minorías raciales al medio universitario, (3) al reforzamiento por medio de la enseñanza de las jerarquías sociales y en particular profesionales y (4) a la exclusión de las cuestiones de justicia económica, social, racial y sexual de los programas de estudios, exclusión que parece favorecer al statu quo [Pickard, Feinman y Feldman, M. J. Frug 1985, Duncan Kennedy 1982].

De 1977 a 1983 (aproximadamente), el movimiento se extiende rápidamente a causa de la adhesión de profesoras y profesores ya establecidos y del reclutamiento de estudiantas y estudiantes (sobre todo en la Harvard Law School) destinados a la carrera académica, sin reacción notable por parte de las corrientes dominantes de las facultades de Derecho. Ello no obstante, la intervención llega, con una eficacia sorprendente para las participantas y participantes, a desestabilizar los metadiscursos corrientes en las facultades, como el de la ortodoxia centrista [compárese Dworkin 1977 y Dworkin 1986] o el del movimiento «Derecho y sociedad» [compárese Seron y Munger y Sarat].

Llegado el caso, los guardianes del orden intelectual se deciden a «contener la ola roja» [Johnson, Schwartz, Carrington]. Varias investigadoras e investigadores ven cómo se les niegan puestos académicos a causa de su asociación con CLS [G. Frug 1987]. Durante este mismo período, CLS, al que se percibe como una escuela «extremista» aunque «seria» en la gama de las tendencias teóricas, se institucionaliza. Sus adherentes de uno y otro sexo ya situados comienzan a beneficiarse de ello, al tiempo que llevan los estigmas correspondiente. Se construye el mito de la Edad de Oro del movimiento, la gente se acomoda a la disipación de las esperanzas de redención, y se vuelve a encontrar, en el interior del CLS, en plena política familiar. Los artículos sobre el tema de CLS sobrepasan en número a los artículos que «hacen» CLS [Duncan Kennedy 1985].

La red es entonces sede de un debate entre «racionalistas» e «irracionalistas». Los racionalistas heredan las tradiciones «científicas» de la izquierda, sobre todo del marxismo, de la sociología de Weber y de Durkheim, y de la historiografía pragmatista americana. Ellas/ellos 
buscan, si no las leyes de la historia, al menos sus «grandes tendencias». Por el término «política» ellas/ellos entienden, si no la lucha de clases, al menos el juego de fuerzas económicas y sociales a nivel de la nación. Su ideal es una fusión del saber en la acción política sobre un modelo más europeo que americano [Horwitz 1977, Unger 1976, 1986, Holt].

Los irracionalistas practican un nuevo método de análisis doctrinal fuertemente influido por el estructuralismo, pero al mismo tiempo se sitúan en las huellas de la vanguardia cultural, tal como los bohemios, beatniks, hippies y situacionistas, así como en las del marxismo de oposición, el anarcosindicalismo, el existencialismo, el feminismo radical, etc. La voluntad de desestabilización prima sobre la de fundar una escuela sobre premisas coherentes y con una jerarquía interna. Ellas/ellos aceptan los límites de una política de facultad, y desconfían de las pretensiones de grandeza que encuentran en las aspiraciones a la cientificidad y al poder de la intelligentsia izquierdizante [Gabel y Kennedy, Freeman 1981, Kelman 1984, C. Dalton 1983].

El artículo antes citado de Rick Abel sobre CLS es un documento típicamente racionalista del período de transición entre la primera y la segunda fase de la intervención de CLS. El presente artículo es un producto típico de la tendencia irracionalista responsable de esta transición. La base material de la transición es el rápido crecimiento del número de mujeres y de miembros de las minorías raciales entre los estudiantes de Derecho, y a continuación en el interior del cuerpo profesoral.

La segunda fase comienza hacia 1983 con el «rights debate» (debate sobre los derechos de la persona). Según la corriente dominante de CLS, el modo como se emplea el concepto de derechos de la persona en las teorías liberales y progresistas sería incoherente y alienante [Gabel y Kennedy, Gabel 1984, Tushnet 1984, Olsen 1984, Paull. A través del debate, tres grupos contestan a la vez la validez de esta crítica, pensando que lo que importa es «salvar» o «reconstruir» un programa basado en los derechos de la persona, y la tendencia de CLS a ignorar las cuestiones que les afectan. Los grupos reúnen: (1) a las abogadas y abogados activistas de izquierda que se interesan por las cuestiones teóricas [Sparer, Lynd]; (2) a las profesoras feministas que quieren superar el feminismo liberal (Schneider 1986a, Minow 1987]; (3) a los miembros de minorías raciales que formulan una base teórica para una nueva ola de acción militante contra el racismo [Williams, Delgado, Crenschaw 1988].

Al mismo tiempo que ellas/ellos provocan el «rights debate», las mujeres blancas y los miembros de las minorías raciales someten a la red CLS a una crítica rigurosa respecto a los restos de las jerarquías sexuales y raciales que afectan a su vida interna [Menkel-Medow, Goldfarb, H. Dalton, Bracamonte]. 
Hoy (1992), CLS está en una tercera fase, caracterizada por: (1) La llegada a escena de una nueva generación de adeptos que se interesan por los debates europeos sobre el post-modernismo y el post-estructuralismo y por la teoría literaria americana post-modernista, feminista y antirracista [David Kennedy 1985, Peller 1985, Boyle, Schlag, Balkin, Thomas 1992a, Ashe, Coombe, Berman 1988, 1992]. (2) El trasvase de la fuente de energía y de innovación de CLS a una nueva sede: la de la intersección de CLS con el feminismo radical [Schneider 1986b, West, Keller, White, Duncan Kennedy 1992], la «critical race theory» [Austin 1988, 1989, Cook, Crenshaw 1991, Torres, Peller 1990], y las teorías críticas de la orientación sexual [Danielson, Thomas], y del «postcolonialismo» [Abu-Odeh, Engle].

(3) La división y la dispersión de la antigua red, una minoría importante de la cual rechaza a la vez el post-modernismo y las preocupaciones de los «Others» («Otros» de pertenencia multicultural) prefiriendo, a la vida a veces demasiado conflictiva de la red, el desarrollo de sus ideas en las revistas académicas.

(4) La concentración de la energía de las facciones de izquierda en las facultades en cuestiones de reclutamiento y de promoción de profesoras y profesores, con la esperanza de cambiar las prácticas mediante el cambio de personal, y el cuasi abandono de la estrategia de confrontación cultural.

(5) La emergencia de un debate sobre la posibilidad de coexistencia de las actitudes hipercríticas del antiguo CLS, de Derrida y de Foucault, con las aspiraciones programáticas de los activistas multiculturales, debate que recuerda al que tuvo lugar entre racionalistas e irracionalistas [M. J. Frug 1992, Minow 1992].

(6) El reparto consciente y deliberado del poder en la organización de las actividades de la red, tal como el congreso de abril de 1992 en Boston, organizado por los «crit networks» (red de redes), que reunió a CLS, «fem-crits» y «race-crits», y dio nacimiento a una nueva red de las lesbianas y los homosexuales.

Si sucediera que la red CLS dejara de existir como entidad distinta del movimiento general de la izquierda en las facultades de Derecho, ¿cómo hacer el balance de sus quince años de actividad? He aquí una respuesta preliminar desde el punto de vista de un "antiguo militante»: CLS no ha elaborado un nuevo programa político y no ha influido ni en la vida política americana ni en la vida interna de la profesión jurídica. No ha transformado la enseñanza del Derecho, aunque haya establecido enclaves y sigan estando en curso experiencias en esta dirección. Ha sido un factor importante (quizá determinante) en la formación de una izquierda jurídica institucionalizada, fenómeno nuevo 
en los Estados Unidos, que surgió en un momento en el que en otras disciplinas fracasaban intentos semejantes, y en un ambiente político de los más desfavorables. Ha dotado a este movimiento de una nueva crítica interna de la razón jurídica, de un saber sesentayochista, de una orientación teórica/irónica/avanzada, y de una política interna de coalición multicultural. «No un mapa, pero sí quizás una brújula».

\section{BIBLIOGRAFÍA}

Richard Abel. «Why does the A.B. A. Promulgate Ethical Rules?», 59 Tex. L Rev. 639 (1981).

Lama abu-Odeh, «Post-Colonial Feminism and the Veil: Considering the Differences», 26 New Eng. L Rev. (1992).

Marie Ashe, «Zig-Zag Stitching and the Seamless Web: Thoughts on «Reproduction» and the Law,» 13 Nova L Rev. 355 (1989).

Regina Austin, «Employer Abuse, Worker Resistance, and the Tort of intentional infliction of Emotional Distress,» 41 Stan. L Rev. 1(1988).

Regina Austin, «Sapphire Bounde,» 1989 Wis. L Rev. 539 (1989).

Joel Bakan, «Constitutional Arguments: Interpretation and Legitimacy in Canadian Constitutional Thought,» 27 Osgood Hall L.J. 123(1989).

Jack Balkin, «The footnote,» 83 N W. U. L Rev. 275 (1988)

Nathaniel Berman, «Sovereignty in Abeyance: Self- Determination and International Law», 7 Wis, Int'l L.J. 51 (1988).

Nathaniel Berman, «Modernism, Nationalism, and the Rhetoric of Reconstruction», Yale J. Law \& Humanities 351 (1987).

Guyora Bindes, «On critical Ley al Studies and gursilla Worfarl», 78900. L.J. 1 (1987)

Pierre Bourdieu, Homo Academicus (Paris: Editions de Minuit, 1984).

James Boyle, «The Politics of Reason: Critical Legal Theory end Local Social Thought», 133 U. Pa. L. Rev. 685 (1985).

José Bracamonte, Toreward: Minority Critiques Of the critical Legal Studies Movement», 22 Harv. CR-CL L. Rev. 297 (1987).

Paul Carrington, «Of law and the River,» 34 J. Legal Educ. 222 (1984).

Ruth Colker, «Anti-Subordination Above All: Sex, Race, and Equal Protection», 61 N. Y. U. L. Rev. 1003 (1985).

Anthony Cook, «Beyond Critical Legal Studies: The Reconstructive 
Theology of Dr. Martin Luther Ding. Jr.», 103 Harv. L. Rex, 985 (1990).

Rosemary Coombe, «Room for Manoauver: Toward a Theory of Practice en

Critical Legsl Studies», 14 Law \& Soc. Inquiry 69 (1989).

Kimberte Crenshaw, «Race, Reform, and Retrechment: Transformation and

Legitimation in Antidiscrimination Law», 101 Harv. L. Rev. 1331 (1988).

Kimberte Crenshaw, «Mapping the Margings: Identity Polities, intersectionality and Violence Against Women,» 43 Sten. L. Rev. 1241 (1991).

Clare Dalton, «An Essay in the Deconstruction of Contract Doctrine», 94 Yale

L.J. 999 (1985).

Clare Dalton, «book review». 6 Harv. Wom. L.J. 229 (1983). Reviey The

Politics of Law: A progressive Critique (D. Kairys ed. 1982).

Harion Dalton, The Clouded prism,» 22 Harv. C.R.-C.L. L. Rev. 435(1987).

Dan Danielson, «Representing identities: Legal Treatament of Pregnancy and

Homosexuality,» 26 New Eng. L. Rev. (1992).

Richard Delgado, «The Ethereal Scholar. Does Critical Legal Studies Have What

Minorities Want?», 22 Harv. C.R.-C.L. L. Rev. 301 (1987).

Ronald Dworkin, Taking Rights Seriously (Cambridge: Harvard Univ. Press, (1977).

Ronald Dworkin, Law's Empire (Cambridge: Belknap Press, (1986).

Karen Engle, International Human Rights and Feminism: When Discourses Meet», 13 Mich. J. int'1 L. 517 (1992).

Jay Feinman and Mark Feldman, «Pedagogy and Politics,» 73 Geo. L.J. 875 (1985).

Allan Freeman, «Legitimizing Racial Discrimination Through Antidiscrimination

Law. A Critical Review of Supreme Court Doctrine», 62 Minn.:. Rez. 1049 (1978).

Allan Freeman, «Truth and Mystification in legal Scholarship,» 90 Yale L.J. 1229 (1981).

Gerald Frug, «Book Review, «22 Harv. CR-CL L. Rev. 665 (1987). (Reviewing

E. Schrecker, No Ivory Tower McCarthyism and the Universities (1986).

Mary Joe Frug. «A Postmodem Feminist Legal Manifesto (an unfinished draft)»,

105 Harv. L. Rev. 1045 (1992).

Mary Joe Frug, «Re-Reading Contracts: A Feminist Anaysis of a Contracts

Casebook», 34 Am. U.L. Rev. 1065 (1985). (Reviewing J. 
Dawson, W Harvey and S. Henderson, Cases and comment on contract (4th ed., 1982)).

Peter Gabel «The Phenomonologie of Rights-consciousness and the pact of the Withdrawn Selves», 62 tex. L. Rev. 1563 (1984).

Peter Gabel, «Book Review», 91 Harv. L. Rev. 302 (1977). (Reviewing R. Dworkin, Taking Rights Seriously (1977)).

Peter Gabel and Paul Harris, «Building Power and Breaking Images: Critical Legal Theory and the Practice of Law,» N. Y. U. rev. L. \& Soc. Change 369 (1982- 1983).

Peter Gabel and Duncan Kennedy, «Roll Over Beethoven», 36 Stan. L. Rev. 1 (1984).

Phyllis Goldfarb. «From the Worlds of 'Others' and Feminist Responses to Critical Legal Studies». 26 New eng. L. Rev. 683 (1992).

Robert Gordon, «New Developmentes in Legal Theory», in The Politics of Law. A Progressive Critique 281 (D. Kairys ed.) (New York Pantheon Books, 1982).

Robert Gordon, «Critical Legal Histories», 36 Stan. L. Rev. 57 (1984).

Robert Gordon, «Unfreezing Legal Reality: Critical Approaches to Law», 15 Fia.

State L. Rev. 195 (1987).

Wythe Holt, «Tift», 52 Geo. Wash. L. Rev. 281 (1984).

Morton Horwitz, The Transformation of American Law, 1780-1860 (Cambridge: Harvard Univ. Press. 1977).

Morton Horwitz, The Transformation of American Law, 1870-1960: The Crisis of Legal Orthodoxy (Oxford: Oxford Univ. Press. 1992);

Allan Hutchinson and Andrew Petter, «Private Rights/Public Wrongs: The Liberal Lie of the Charter», 38 U. Toronto L.J. 278 (1988).

Philip Johnson, «Do You Sincerely Want to be Radical?», 36 Stan. L. Rev. 247 (1984).

Al Katz, "Studies in Boundary Theory: Three Essays in Adjudication and Politics,» 28 Buffalo L. Rev. 383 (1979).

Susan Keller, «Powerless to Please: Candida Royalle's Pornography for Women», 26 New Eng. L. Rev. (1992).

Mark Kelman, «Interpretive construction in the Substantive Criminal Law,» 33 Stan.

L. Rev. 591 (1981).

Mark Kelman, A Guide to Critical legal Studios, Cambridge: Horward Unis. Press, 1987.

Mark Kelman, «Trashing», 36 Stan. L. Rev. 293 (1984).

David Kennedy, «Spring Break» 63 Tex. L. Rev. 1377 (1985).

David Kennedy, «Primitive Legal Scholarship», 27 Harv. Int’1 L.J. 1(1986). 
Duncan Kennedy. «Form and Substance in Private Law Adjudication», 89 Harv. L. Rev. 1685 (1976).

Duncan Kennedy, «The Structure of Blackstone's Commentaries», 28 Buffalo L. Rev. 205 (1979).

Duncan Kennedy, «Legal Education and the Reproduction of Hierarchy», 32 J. Legal Educ. 591 (1982).

Duncan Kennedy, «Psycho-Social CLS: A Comment on the Cardozo Symposium», 9 Cardozo L. Rev. 1013 (1985).

Duncan Kennedy, «A Semiotics of Legal Argument», 42 Syracuse L. Rev. 75 (1991).

Duncan Kennedy, «Sexual Abuse, Sexy Dressing and Eroticization of Domination» 26 New Eng. L. Rev. (1992).

Kart Klare, «Judicial Deradicalization of the Wagner Act and the Origins of Modern Legal Consciousness, 1937-1941,» 62 Minn. L. Rev. 265 (1976).

Kart Klare, «Law-Making as Praxis,» Telos, Summer 1979, 123; Deborah Livingston, «Round and Round the Bramble Bush: From Legal Realism to Critical Legal Scholarship», 95 Harv. L. Rev. 1669 (1982).

Staughton Lynd, «Communal Rights», 62 Tex L. Rev. 1417 (1984).

Patrick Macklem, Boak Review 41 U. Toronto L.J. A. 114 (1991). (Reviewing A. Hutchinson, Dwelling or The Threschold: Critical Essags in Modern Legal Thought (1963).

Carrie Menkel-Meadow, «Feminist Legal Theory, Critical Legal Studies, and Legal Education or The Fem-Crits Go to Law School», 38 J. Legal Educ. 61 (1988).

Elizabeth Mensch, «Book Review», 33 Stan. L. Rev. 753 (1981). (Reviewing P. Atiyah, The Rise and Fall of Freedom of Contract (1979)).

Martha Minow, «Interpreting Rights: An Essay for Robert Cover», 96 Yale L.J. 1860 (1987).

Martha Minow, «Incomplete Correspondence: An Unsent Letter to Mary Jos Frug». 105 Harv. L.Rev. 1096 (1992).

John Nockleby. «Tortious Interference with Contractual Relations in the Nineteenth Century: The Transformation of Property, Contract and Tort», 93 Harv. L. Rev. 1510 (1980).

Frances Olsen, «The Family and the Market: A Study of Ideology and Legal Reform», 96 Harv. L. Rev. 1497 (1983).

Frances Olsen, «Statutory Rape: A Feminist Critique of Roghts Analysis», 63 Tex. L. Rev. 387 (1984).

Frances Olsen, «The sex of Law,» in The Politics of Law: A 
Progressive Critique (D. Kairys ed. New York: Pantheon Books. rev. ed. 1990).

Jeremy Paul, «Book Review,» 88 Mich L. Rev. 1622 (1990). (Reviewing J. Waldron, The Right To Private Property (1988)).

Gary Peller, «The Metaphysics of American Law», 73 Callf. L. Rev. 1152 (1985).

Gary Peller, «Race Consciousness», 1990 Duke L.J. 758 (1990).

Rudolph Peritz, «The 'Rute of Reason' in Antit ust: Property Logic in Restraint of competition», 40 Hastings L. Rev. 285 (1989).

Toni Pickard, «Experience as Teacher: Discovering The Politics of Law Teaching», 33 U. Toronto L.J. 279 (1983).

Austin Sarat, «Legal Effectiveness and Social Studies of Law: On the Unfortunate Persistance of a Research Tradition», 9 Legal Stud. Forum 23 (1985).

Pierre Schiag. «Normative and Nowhere To Go». 43 Stan. L. Rev. 167(1990).

John Schiegel, «Notes Toward an Intimate, Opinionated, and Affectionare History of the Conference on Critical Legal Studis», 36 Stan. L. Rev. 391 (1984).

Elizabeth Schnelder, "The Dialectic of Rights and Politics: Perspectives from the Women's Movement», 61 N.Y.U.L. Rev. 589 (1986). [«1986a»]; Elizabeth Schneider, «Describing and Changing: Women's Self-Defense Work and the Problem of Expert Testimony on Battering,» 9 Wom. Rts. L. Rep. 195 (1986) [«1986b»].

Louis Schwartz, «With Gun and Camera Through Darkest CI-SLand,» 36 Stan. L. Rev. 247 (1984).

Carroll Seron and Frank Munger, «Critical Legal Studies Versus Critical Legal Theory: A Comment on Method», 6 Law \& Poly 257 (1984).

William Simon, «Legality, Bureaucracy, and Class In the Welfare System», 92 Yale L.J. 1198 (1983).

Joseph Singer, «The Legal Rights Debate in Analytical Jurisprudence from Bentham to Hohfeld», 1982 Wis. L. Rev. 975 (1982).

Edward Sparer, «Fundamental Human Rights, Legal Entitiements, and the Social Struggle: A Friendly Critique of the Critical Legal Studies Movement», 36 Stan. L. Rev. 509 (1984).

Robert Steinfeld, The Disappearance of Indentured Servitude and the Invention of Free Labour in the United States (Chapel Hill: Univ. of North Carolina Press, 1991):

Katherine Stone, «The Post- War Paradigm in American Labor Law», 90 Yale L.J. 1509 (1981). 
Kendall Thomas, «'Rouge et Noire' Re-read: A Popular Constitutional History of the Angela Herndon Case», S. Cal. L. Rev. (1992) [«1992a»].

Kendall Thomas, «Beyond the Privacy Principle», 92 Colum. L. Rev. 501 (1992) [«1992b»].

Gerald Torres, «Local Knowiedge, Local Color: Critical Legal Studies and the Law of Race Relations», 25 San Diego L. Rev. 1043 (1988).

David Trubek, «Where the Action is: Critical Legal Studies and Empiricism», 36 Stan. L. Rev. 575 (1984).

Mark Tushnet, Red, White and Blue: A Critical Analysis of Constitution Law (Cambridge: Harvard Univ. Press. (1988).

Mark Tushnet, «An Essay on Rights», 62 Tex. L. Rev. 1363 (1984).

Mark Tushnet, «Critical Legal Studies: A Political History», 100 Yale L.J. 1515 (1991).

Mark Tushnet, «Book Review», 68 Cornell L. Rev. 281 (1983). (Reviewing L. Tribe, Marxism and The Law (1982)).

Roberto Unger, Knowedge and Politics (New York: Free Press, (1975).

Roberto Unger, Law in Modern Society: Toward a criticism of Social Theory (New York: Free Press, 1976).

Roberto Unger, The Critical Legal Studies Movement (Cambridge: Harvard Univ. Press, 1986).

Kenneth Vandevelde, «The New Property of the Nineteenth Century: The Devalopment of the Modern Concept of Property», 29 Buffalo L. Rev. 325 (1980).

Robin West, «Jurisprudence and Gender», 55 U. Chi. L. Rev. 1 (1988).

Lucie White, «Subordination, Rhetorical Survival Skilis, and Sunday Shoes: Notes on the Hearing of Mrs. G.,» 38 Buffalo L. Rev. 1 (1990).

Patricia Williams, The Alchemy of Race and Rights (Cambridge: Harvard Univ. Press, 1991). 
$\triangle$

DOXA-11 (1992) 